\title{
NITROGEN AND CARBON ANOMALIES IN OB SPECTRA
}

\author{
N. R. WALBORN \\ Cerro Tololo Inter-American Observatory, La Serena, Chile
}

\begin{abstract}
The following new OBN and OBC stars have been found: HD 75860, BC 2 Iab; HD 104565, OC 9.7 Ia; HD 123008, ON 9.7 Iab; and HD 150574, ON 9 III(n). Examples of morphologically more moderate $\mathrm{CNO}$ anomalies in $\mathrm{OB}$ spectra are also discussed; it is concluded that the O9-B0 7 supergiants of the Orion Belt and NGC 6231 (the nuclear cluster of Scorpius OB1) are systematically nitrogendeficient. One possible explanation of these nitrogen-deficient supergiants in sub-associations is that they may have formed from nitrogen-deficient clouds; another could be that the more numerous morphologically normal supergiants show the effects of mixing in their spectra, while the nitrogendeficient ones are relatively younger or have not yet been red supergiants. No red supergiants are found in Sco OB1, whereas NGC 3293 contains two B0 supergiants with normal CNO spectra together with a red supergiant. The paper will appear in the Astrophysical Journal, April 15, 1976.
\end{abstract}

\section{DISCUSSION}

Garrison: Your system depends very heavily on ratios of the He with $\mathrm{Si}$. How confident are you that there are no $\mathrm{Si}$ anomalies and that all differences are due to $\mathrm{CNO}$ anomalies?

Walborn: The spectral types depend mostly upon ratios of successive stages of ionization of $\mathrm{He}$ and $\mathrm{Si}$. The luminosity classes depend upon ratios of $\mathrm{Si}$ to He; however, the whole spectrum is inspected for consistency. For instance, if $\mathrm{Si} / \mathrm{He}$ indicated a supergiant class, but the Balmer lines had wings, a discrepancy would be obvious; such cases are not seen. As in the classical MK system, the classification is an empirical procedure, whose validity is checked later by the calibrations. The He-Si types agree systematically with MK types for stars in common, and the calibration results are satisfactory.

Houziaux: It is remarkable that such a refined classification can be obtained for very hot objects since in your spectral range you observe, you collect a very small fraction of the total energy of the star. I am wondering if it would not be wise to wait now for satellite UV data, as the spectral differences should be much more spectacular in this wavelength range, the Planck function being much more sensitive to differences in temperature between 1200 and $2000 \AA$ than it is in the 3800-5000 $\AA$ region.

Walborn: The absolute-magnitude and effective-temperature calibrations support the validity of the classification in the blue-violet spectral region. The more highly refined this classification can be made, the more valuable will be the eventual comparison with far-UV results. 